\title{
Operation Methods for Grid-connected PV system for Voltage Control in Distribution System - A Review
}

\author{
Ms. Priya Patel M.Tech Student, CGPIT, Bardoli Surat, Prof.Jignesha Ahir, Prof. Arjun Jariwala Assistant Professor, \\ CGPIT, Bardoli Surat, India.
}

\begin{abstract}
PV solar systems employ inverters to transform dc power from solar panels into ac power for injecting into the power grids. Inverters that perform multiple functions in addition to real power production are known as "smart inverters". This paper presents a novel control of PV inverter as a dynamic reactive power compensator-STATCOM. This "smart PV inverter" control enables a PV solar inverter to operate in three modes - i) Full PV, ii) Partial STATCOM, and iii) Full STATCOM, depending upon system needs.
\end{abstract}

Keywords-Photovoltaic; Static Synchronous Compensator.

\section{INTRODUCTION}

Environmental concerns such as pollution and limited resources of gas and fossil fuels have caused a surge of interest in renewable energy in recent years. One of the major renewable energy sources in the world is the photovoltaic system (PV) which converts solar power to electrical power.

In Full PV mode, the inverter performs only real power production based on solar radiation. In Partial STATCOM mode, the controller uses the remaining capacity of the inverter for voltage control, power factor correction and reactive power control. The Full STATCOM mode is invoked in emergency scenarios, such as faults, or severe voltage fluctuations. In this mode, the real power production is shut down temporarily and the entire inverter capacity is utilized for voltage regulation for providing critical support to the power system.

\section{CONCEPT OF SMART PV INVERTER CONTROL AS STATCOM (PV-STATCOM)}

As STATCOM is a dynamic reactive power compensator based on voltage source converter (VSC), whereas a conventional PV system requires a VSC for converting DC power to AC power. On the other hand, a STATCOM is a device to exchange reactive power whereas a PV system generates active power. Therefore, the combination of these two concepts can support both active and reactive power. A new technology has been proposed for utilizing a PV solar system inverter as a STATCOM. The power output of a PV system during a typical sunny day is shown in Figure 1.

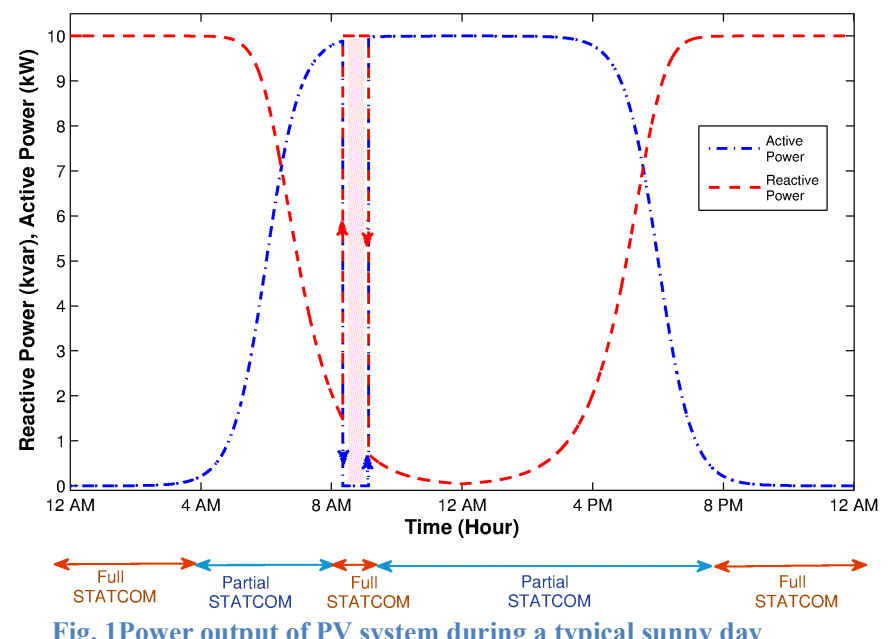

Fig. 1Power output of PV system during a typical sunny day

\section{DESIGN OF SMART PV INVERTER CONTROL FOR THE STUDY SYSTEM}

Smart PV inverter control implies a multifunctional control of a PV inverter in addition to its prime purpose of real power generation. The smart PV inverter is a Voltage Source Converter (VSC) system with the capability of exchanging (injecting or absorbing) both active power and reactive power. The structure of the controller is based on controlling active power and reactive power through phase angle and voltage amplitude, respectively. In $d-q$ reference frame, due to decoupled control, $d$ - axis current loop controls active power and $q$-axis current control loop controls reactive power. The references value for the both current control loops are defined based on the smart inverter operation mode and control objectives.

\section{A. abc to dq TRANSFORM}

In $a b c$-frame and $\alpha \beta$-frame, the reference, feedback, and feed-forward signals are sinusoidal functions of time. In $d q$-frame sinusoidal signals are transformed to equivalent DC signals which are independent of time variation. Consequently, the compensator can be designed with better dynamics and is capable of having zero steady-state error by applying an integral term. The smart PV inverter controller is modeled in a synchronously rotating $d-q$ reference frame to achieve better 


\section{Asian Journal of Convergence in Technology} ISSN NO: 2350-1146 I.F-5.11

transient and steady-state performances. Figure 2 shows the phase diagram of a vector in $a b c$-frame and $d q$-frame. In Figure 2, the vector $f(t)$ rotates with time-variant frequency $\omega(t)$ in $a b c$-frame. The phase difference between the rotating vector $f(t)$ and stationary axes in $a b c$-frame is defined by $\theta(t)$ whereas $\theta_{0}$ indicates the initial phase angle. To achieve nontime-variant parameters, $d q$-frame needs to rotate with same frequency $\omega(t)$. In Figure 2, $\phi(t)$ is phase difference between $a b c$-frame and $d q$ - frame whereas $\rho(t)$ is phase difference between rotating vector and rotating $d q$-frame.

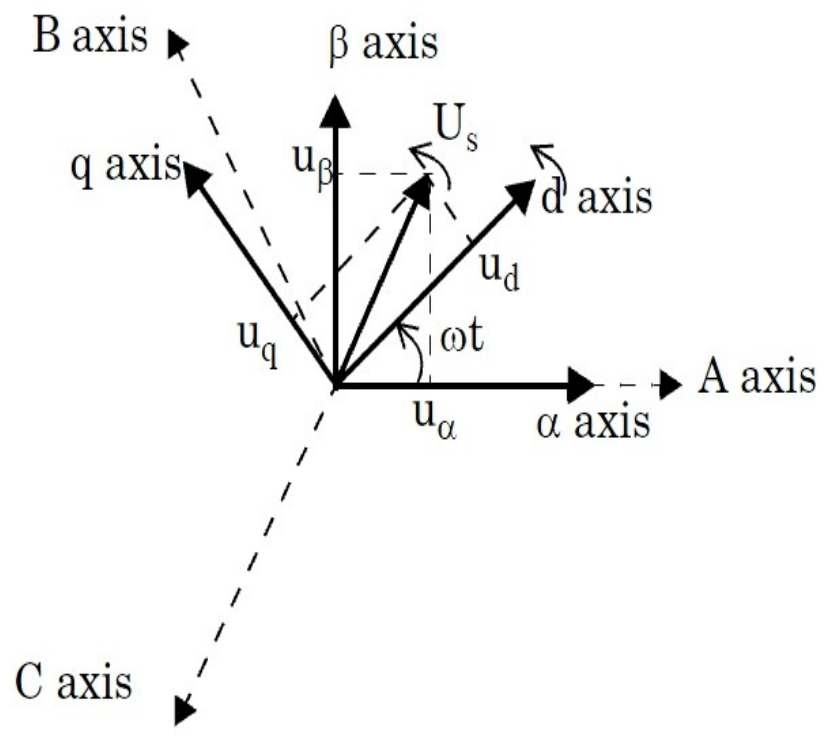

Fig. 2 abc to dq transformation

B. PLL Design

The PCC voltages can be expressed as:

$$
f_{d}+j f_{q}=\hat{f} e^{j\left(\omega t+\theta_{0}\right)} e^{-j \omega t}=\hat{f} e^{j \theta_{0}}
$$

The above equation confirms that both $d$ and $q$ components are DC quantities. In other words, by extracting the frequency of the signal and using in $a b c$ to $d q$ transformation, the $d$ and $q$ components can be achieved as DC quantities.

\section{Design of Current Controller}

$$
\begin{aligned}
& L \frac{d i_{i d}}{d t}+R i_{i d}=u_{d} \\
& L \frac{d i_{i q}}{d t}+R i_{i q}=u_{q}
\end{aligned}
$$

The current components and control signals can be considered as the controller inputs and controller output, respectively. For achieving a proper modulating signal, the

\section{Volume V Issue I}

feed-forward terms are added to the controller signals based on

$$
\begin{gathered}
M_{d}=\frac{2}{V_{D C}}\left(u_{d}-L_{f} \omega_{0} i_{i q}+V_{p c c-d}\right) \\
M_{q}=\frac{2}{V_{D C}}\left(u_{q}+L_{f} \omega_{0} i_{i d}+V_{p c c-q}\right)
\end{gathered}
$$

and

Hence, the uncompensated open-loop transfer functions of current control are:

$$
\begin{aligned}
& \frac{u_{d}}{i_{i d}}=\frac{1}{L_{f} s+R_{f}} \\
& \frac{u_{q}}{i_{i q}}=\frac{1}{L_{f} s+R_{f}}
\end{aligned}
$$

The above two equations reveal that the dynamic equations of current in $d-q$ frame are stable. Thus, a PI controller can be used to make the steady-state error zero and decrease the response time. To reduce the response time, it needs to move the transfer function pole farther from origin of the real-imaginary coordinates.
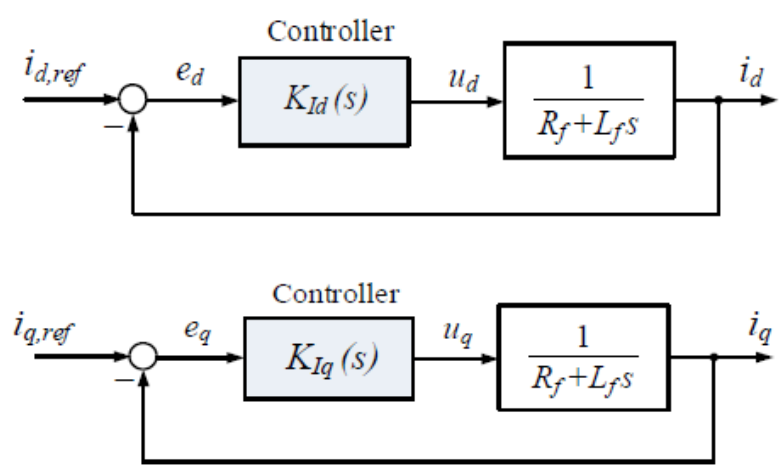

Fig. 3 current loop with PI controller

a) d-Component control loop b) q-Component control loop

\section{OPERATION MODE SELESCTOR OF THE SMART PV INVERETR CONTROL}

During daytime, the smart inverter controller operates as a conventional PV system and generates active power. If control objectives require exchange of reactive power to either regulate the PCC voltage or provide power factor correction, the controller uses the remaining capacity of the inverter for exchanging reactive power. This mode of operation is called "Partial STATCOM Mode". In this partial mode, the priority of the smart inverter is the generation of the active power and 


\section{Asian Journal of Convergence in Technology ISSN NO: 2350-1146 I.F-5.11}

then exchange of reactive power with remaining inverter capacity. But during transients or faults the grid needs more reactive power support to maintain the voltage within an acceptable range. Consequently, the smart PV control disconnects the DC switches of solar panel and transforms the PV inverter to full STATCOM to exchange reactive power with the full capacity of the inverter. This mode of the smart PV control is termed "Full STATCOM Mode". It should be noted that in full STATCOM mode, voltage regulation is considered as the sole control objective. The smart PV inverter autonomously determines its operating mode and prioritizes between active power generation and reactive power exchange based on the system requirements, nature of transient/disturbance, time of the day and remaining inverter capacity.

Figure-4 shows the flowchart of the smart PV inverter control to define the operation mode. During daytime, the inverter can act as a full PV, full STATCOM or partial STATCOM. Initially, the inverter operates as a PV inverter in conventional real power generation mode. Assume the PV panel is connected to the inverter and the PCC voltage suddenly violates the acceptable range. Based on the control algorithm, the controller immediately disconnects the switches at its DC side and converts the PV system to full STATCOM. During full STATCOM mode, the control objective is set to Voltage Regulation (VR) automatically and it cannot be changed to power factor correction (PFC). When fault is cleared, the reactive power output of the inverter is negligible. Hence, the controller realizes the fault status by checking DC switch status and reactive power output of the inverter. After fault has been cleared, the controller reconnects the solar panel to the inverter and operates again in full PV mode.

When the solar panel is connected to the inverter and utility needs to either regulate the voltage within acceptable range or correct the power factor, the remaining inverter capacity is used for exchanging reactive power. In other words, the reactive power requirement is limited by:

$$
Q_{\max }=\sqrt{S^{2}-P^{2}}
$$

where $S$ is apparent power of the inverter, $P$ is actual power of the inverter. During nighttime, the DC switch disconnects the solar panel from the inverter. Therefore, the control mode is full STATCOM. But the voltage changes can occur transiently or in steady-state. When PCC voltage is in acceptable range, the control objective can be either voltage regulation or power factor correction. But when the voltage violates the defined range due to transient events, e.g., Temporary Over Voltage TOV), the controller objective is set to voltage regulation and operates with its full capacity. Same as daytime, the controller realizes the fault has been cleared by reactive power output and previous state of its operation.

\section{Volume V Issue I}

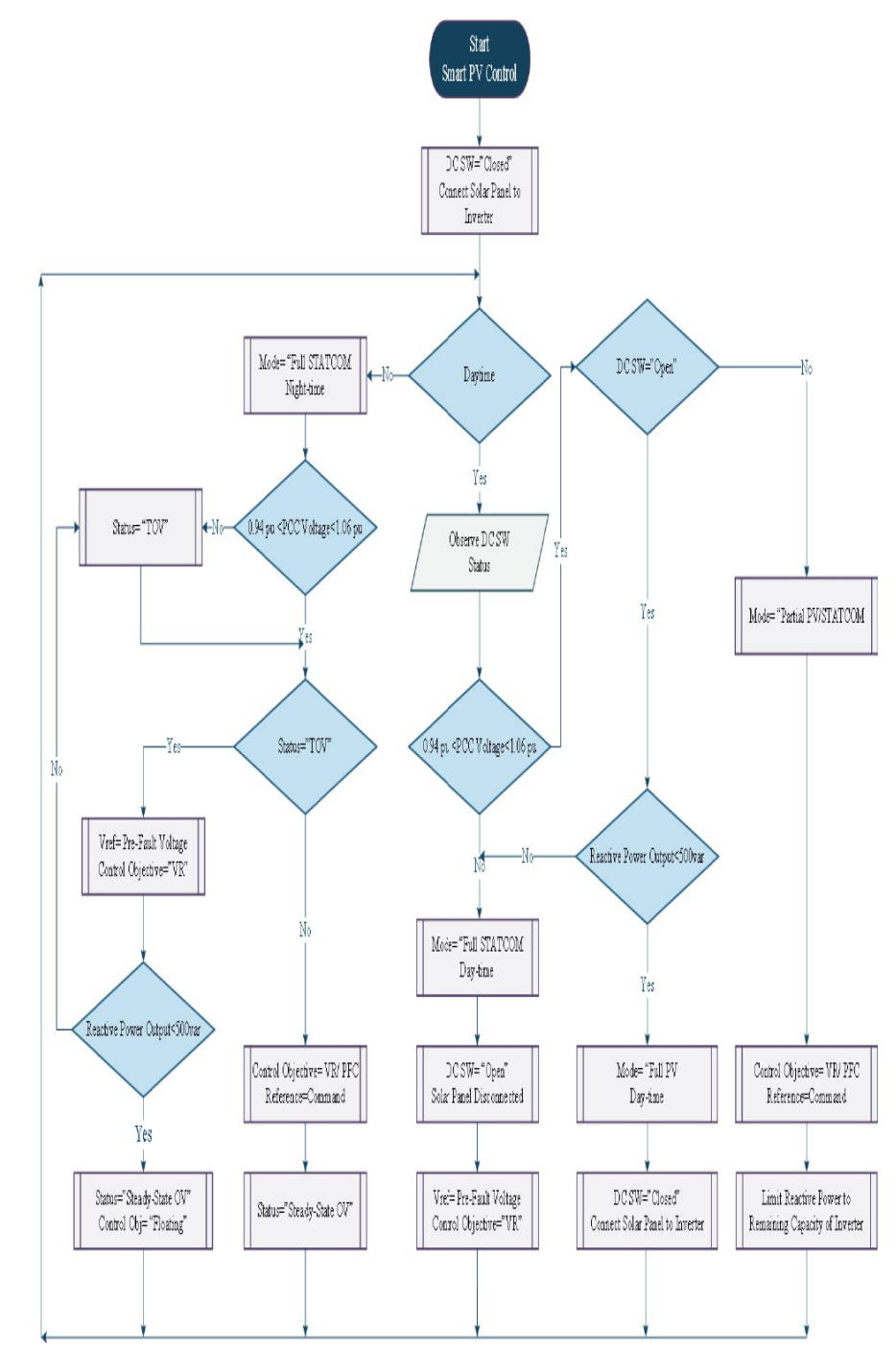

Fig. 4Flowcharrt of the smart PV inverter operating modes

\section{Conclusion}

The modeling of different components of a typical radial distribution system with a PV solar system. The operating principles of the smart PV inverter are presented for both Partial and Full STATCOM modes, for the two control objectives of voltage control and power factor correction. The different inverter components and controller parameters are designed for a 10kVA smart PV inverter system to be implemented in the distribution network of the Bluewater Power, Sarnia. The smart inverter controller includes PLL, $a b c-d q$ transformation block, $d-q$ current controller, DC voltage controller, AC bus voltage controller, mode selector unit, power factor correction unit and PWM unit. The design procedure of each component is described for the study 
system. The controller parameters are designed utilizing classic control theory.

\section{References}

[1] R. K. Varma, S. A. Rahman, A. C. Mahendra, R. Seethapathy, and T. Vanderheide, "Novel nighttime application of PV solar farms as STATCOM (PV-STATCOM)," in 2012 IEEE Power and Energy Society General Meeting, July 2012, pp. 1-8.

[2] R. K. Varma, S. A. Rahman, and T. Vanderheide, "New control of PV solar farm as STATCOM (PV-STATCOM) for increasing grid power transmission limits during night and day," IEEE Transactions on Power Delivery, vol. 30, no. 2, pp. 755-763, April 2015.

[3] R. K. Varma, V. Khadkikar, and S. A. Rahman, "Utilization of distributed generator inverters as STATCOM," Sep. 15 2010, PCT Patent application PCT/CA2010/001419.

[4] JW Smith, W Sunderman, R Dugan, and Brian Seal, "Smart inverter volt/var controlfunctions for high penetration of $\mathrm{PV}$ on distribution systems," in Power Systems Conference and Exposition (PSCE), 2011 IEEE/PES, 2011, pp. 1-6.

[5] F. Katiraei, Agu, x, and J. R. ero, "Solar PV Integration Challenges," Power and Energy Magazine, IEEE, vol. 9, pp. 62-71, 2011. 
